International Journal of Pure and Applied Mathematics

Volume 83 No. 4 2013, 539-548

ISSN: 1311-8080 (printed version); ISSN: 1314-3395 (on-line version)

url: http://www.ijpam.eu

doi: http://dx.doi.org/10.12732/ijpam.v83i4.2

\title{
OPTIMAL LINEAR CONTROL TO PARAMETRIC \\ UNCERTAINTIES IN A MICRO ELECTRO \\ MECHANICAL SYSTEM
}

\author{
Fábio Roberto Chavarette \\ Faculty of Engineering of Ilha Solteira \\ UNESP - Univ Estadual Paulista \\ Departament of Mathematics, GMSINT \\ Avenida Brasil, 56, 15385-000, Ilha Solteira, SP, BRAZIL
}

\begin{abstract}
This paper, a micro-electro-mechanical systems (MEMS) with parametric uncertainties is considered. The non-linear dynamics in MEMS system is demonstrated with a chaotic behavior. We present the linear optimal control technique for reducing the chaotic movement of the micro-electromechanical system with parametric uncertainties to a small periodic orbit. The simulation results show the identification by linear optimal control is very effective.
\end{abstract}

AMS Subject Classification: 26A33

Key Words: linear optimal control, chaos control, micro electro mechanical system

\section{Introduction}

Numerous design methodologies exist for the control design of nonlinear system. These include any of a huge number of linear design techniques $[1,2]$, used in conjunction with gain scheduling [3, 4]; nonlinear design methodologies 
include Jacobian linearization, recursive backstepping, feedback linearization, gain scheduling, sliding mode control, and adaptative control [5].

Nonlinear control systems problems consist in stabilize the chaotic dynamic of a system to an equilibrium point or a periodic orbit or to drive to a specific reference trajectory. Lately, a significant interest in control of the nonlinear systems, with chaotic behavior, has been observed and many of the techniques have been discussed in literature [6]-[14]. Among strategies of chaos control with feedback, the most popular is the Ott-Grebogi-York (OGY) method [6]. This method uses the Poincare map of the system. Recently, a methodology, based on the application of the Lyapunov-Floquet transformation, was proposed by Sinha et al. [7] in order to solve this kind of problem. This method allows directing the chaotic motion to any desired periodic orbit or to a fixed point. It is based on linearization of the equations, which described the error between the actual and desired trajectories.

Another interesting method, first proposed by Pearson [15] and later expanded by Wernli and Cook [16], was independently studied by Mracek and Cloutier [9] and alluded to by Friedland [17]. Use of the State-Dependent Riccati Equation (SDRE) to control nonlinear system has seen increasing application $[9,13]$. Here, we use different approaches, which were proposed by Rafikov and Balthazar in [18]: Dynamic Programming was used to solve the formulated optimal control problems and we will obtain functions that satisfied the Hamilton-Jacobi-Bellman, among the correspondent Lyapunov functions of the proposed model.

The aim of this paper is to propose the application of the Linear Optimal Control [18] to control the chaotic movement of a Micro Electro Mechanical System [20].

We organize the paper as it follows: in Section 2, we demonstrated the mathematical model and we showed of the nonlinear dynamics of the model. In Section 3, we discussed a control design problem for non-linear model. In Section 4, we made the concluding remarks of this paper. Finally, we listed out the bibliographic references which we used in this paper.

\section{Microelectromechanical Model}

In the work [20], was considered the microelectromechanical Systems with periodic parametric excitation, as shown in Fig. 1. The device what consists of a cantilevered beam connected to a torsion bar. Attached to the cantilevered beam are an atomically sharp tip for the probe and comb capacitive transducers 


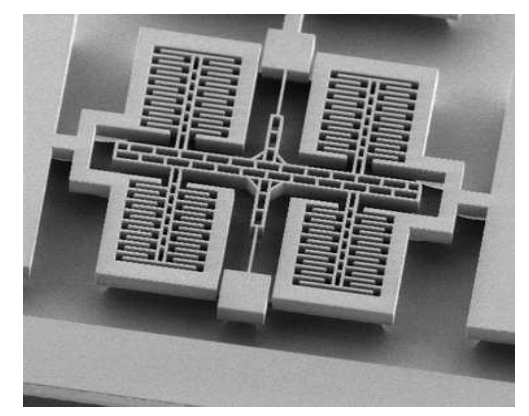

Figure 1: Microelectromechanical torsional oscillator[20].

for sensing and actuation.

The governing equations of system ilustrated in Fig. 1 is proposed in [20], are

$$
\ddot{x}+2 / Q \dot{x}+\left[\omega_{0}+\beta \cos (\omega t)\right] x=0
$$

which is a simple oscillator with a time-varying stiffness term.

The model (1) is a basic model for this class systems [20], its equation has application in the forced motion of a swing, the stability of ships, Faraday surface wave patterns on water, and the behavior of parametric amplifiers based on electronic or superconducting devices.

The Figure 2 illustrate the dynamics behavior of the adopted dynamics model, by using numerical values, for the chosen parameters $a=1 / 3000 ; d=$ $0.046 ; \omega_{0}=0.57 ; n=3 ; \omega=\left(2 \omega_{0}\right) / n$ and $b=-0.000227$.

To consider the effect of the parameter uncertainties on the performance of the controller they are added to the state. The nominal values of parameters are stated above.

The real unknown parameters of system are supposed to be as follows: $\bar{a}=$ $0.000333+0.00001 r(t), \bar{d}=0.046+0.02 r(t), \bar{w}_{0}=55+2 r(t), \bar{n}=2+1 r(t), \omega=$ $\left(2 \omega_{0}\right) / n$ and $\bar{b}=-0.000225+0.000003 r(t)$, where $r(t)$ are normally distributed random functions.

Numerical simulation results are shown in Section 3. This figure shows the robustness of the used methods when the systems parameters have random uncertainties.

The eigenvalues of the system ( 1$)$ are $\lambda_{1}=-0.3695 ; \lambda_{2}=-0.9418$. The eigenvalues $\lambda_{1,2}$ indicates that the system is unstable and the Fig. 2 illustrates the chaotic dynamic $\left(\lambda_{1}=0.4620\right)$ and $\left(\lambda_{2}=0.0870\right)$ of Lyapunov exponent for system (1) in Fig. 3. 

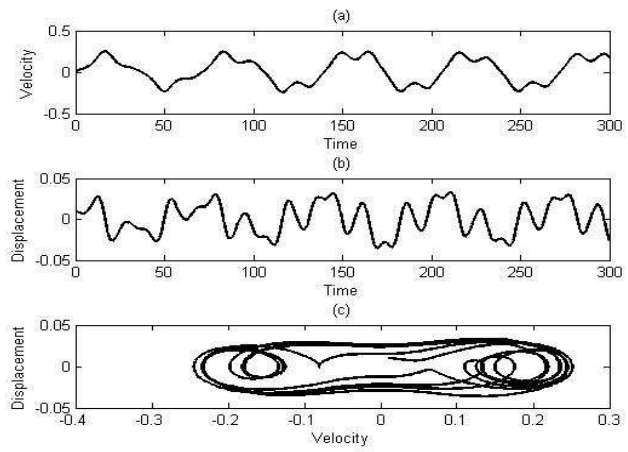

Figure 2: Time History (a) Velocity, (b) Displacement and (c) Portrait Phase.

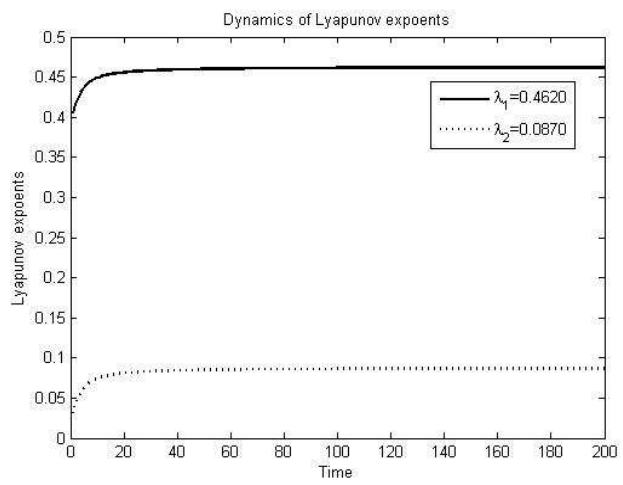

Figure 3: Dynamics of Lyapunov expoents for system

\section{Control Design}

In this section, we applied optimal linear control design for the micro electro mechanical system (Fig. 1), reducing the oscillatory movement to a small stable orbit. Next, we present the theory of the used methodology.

Due to the simplicity in configuration and implementation, the linear state feedback control, it is especially attractive [13].

We remarked that this approach is analytical, and it may use without dropping any non-linear term. Let the governing equations of motion (1), re-written in a state form 


$$
\dot{x}=A x+g(x)
$$

If one considers a vector function $\tilde{x}$, that characterizes the desired trajectory, and taken the control $U$ vector consisting of two parts: tildeu being the feed forward and $u_{f}$ is a linear feedback, in such way that

$$
u_{f}=B u
$$

where $B$ is a constant matrix. Next, one taking the deviation of the trajectory of system (2) to the desired one $y=x-\tilde{x}$, may written as being

It is optimal, in order to transfer the non-linear system (6) from any initial to final state $y\left(t_{f}\right)=0$, minimizing the functional $\tilde{J}=\frac{1}{2} \int_{0}^{\infty}\left(y^{T} \tilde{Q} y+u^{t} R u\right) d t$, where the symmetric matrix $P(t)$ is evaluated through the solution of the matrix Ricatti differential equation

$$
P A+A^{T} P-P B R^{-1} B^{T} P+Q=0
$$

satisfying the final condition $P\left(t_{f}\right)=0$.

In addition, with the feedback control (6), there exists a neighborhood $\Gamma_{0} \subset \Gamma, \Gamma \subset \Re^{n}$, of the origin such that if $x_{0} \in \Gamma_{0}$, the solution $x(t)=0, t \geq$ 0 of the controlled system (4) is locally asymptotically stable, and $J_{\min }=$ $x_{0}^{T} P(0) x_{0}$.Finally, if $\Gamma=\Re^{n}$ then the solution $y(t)=0, t>0$ of the controlled system (4) is globally asymptotically stable.

Using the theorem [17] the dynamic error y can be minimized $(y \rightarrow 0)$.

\subsection{Theorem Linear Optimal Control.}

If there is matrixes $Q$ and $R$, positive definite, $Q$ symmetric, such that the matrix

$$
\tilde{Q}=Q-G^{T}(x, \tilde{x}) P-P G(x, \tilde{x})
$$

is positive definite for the limited matrix $G$, then the linear feedback control

$$
u=-R^{-1} B^{T} P_{y}
$$


is optimal, in order to drive the non-linear system (6) of any initial state to the terminal state

$$
y(\infty)=0
$$

minimizing the functional

$$
J=\int_{0}^{\infty}\left(y^{T} \tilde{Q} y+u^{T} R u\right) d t
$$

where the symmetric matrix $P$ is calculated from the nonlinear Riccati equation:

$$
P A+A^{T} P-P B R^{-1} B^{T} P+Q=0
$$

Next, we will apply this methodology in model (1).

\subsection{Application of the Linear Optimal Control to the System}

The equations (1) describing the system:

$$
\ddot{x}+2 / Q \dot{x}+\left[\omega_{0}+\beta \cos (\omega t)\right] x=U
$$

where the function of control $U$ is defined in the equation (1).

Rewriting the dynamical system (10), in the space-state form, we will obtain:

$$
\begin{array}{r}
\dot{x_{1}}=x_{2}+U \\
\dot{x_{2}}=-2 / Q x_{2}-\left[\omega_{0}+\beta \cos (\omega t)\right] x_{1}
\end{array}
$$

We will obtain $B=\left(\begin{array}{l}1 \\ 1\end{array}\right), y=\left(\begin{array}{c}x_{1}-\tilde{x}_{1} \\ x_{2}-\tilde{x}_{2}\end{array}\right), \tilde{x}=\left(\begin{array}{c}\sin (\pi t) / 50 \\ \cos (\pi t) / 50\end{array}\right), Q=I_{2}$, $A=\left(\begin{array}{cc}0 & 1 \\ -0.04249 & -0.0003\end{array}\right)$ where the controllability matrix $R$ of the system to the pair $[A, B]$ is obtained by $R=[B \mid A B]$.

Thus, $R=\left(\begin{array}{cc}1 & 1 \\ 1 & -0.0428\end{array}\right)$. 

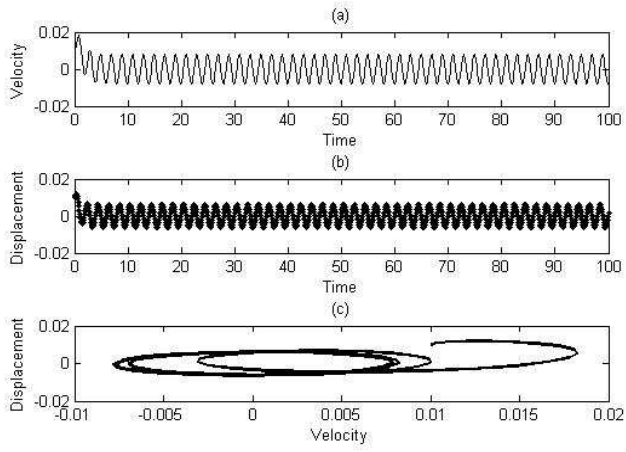

Figure 4: Time History Controlled (a) Velocity, (b) Displacement and (c) Controlled Portrait Phase.

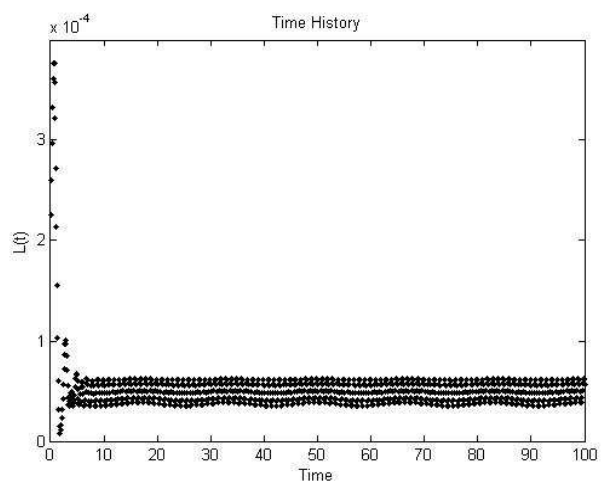

Figure 5: Phase Portrait

Then the Matrix $P(t)$ is done by $P=\left(\begin{array}{cc}1.0218 & -0.0209 \\ -0.0209 & 0.9994\end{array}\right)$ and (an optimal control) $u=1.0008 x_{1}+0.9784 x_{2}$. The trajectories of the system with control may be seen, through Fig. 4. According to the optimal control verification [17], the function (4) is numerically calculated across $L(t)=y^{T} \tilde{Q} y$, where $L(t)$ is defined positive and it is show in Fig. 5.

Figure 6 shown controlled and noncontrolled dynamical behavior Portrait Phase. 


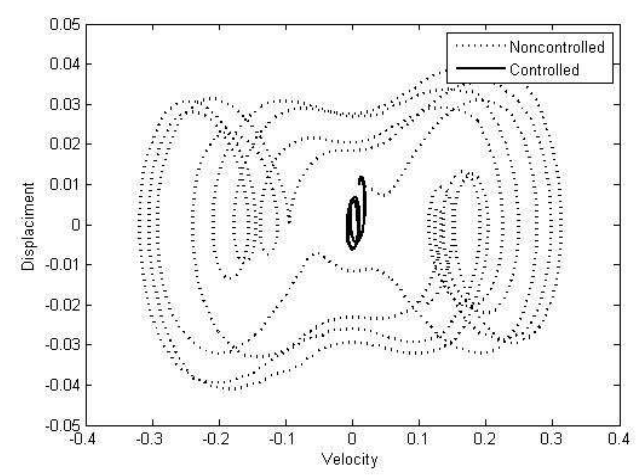

Figure 6: Phase Portrait

\section{Conclusions}

In this work, we proposed the use of an Linear Optimal Control strategy applied to Micro-electro-mechanical system with parametric uncertainties.

A torsional oscillator system is modeling using the Micro-electro-mechanical system. In comparing of numerical results of controlled system with the non controlled system (Fig. 6) we can verify that control orbit generated by Linear Optimal Control (Fig. 6) has small diameter.

The technique control reducing the chaotic movement of the MEM systems to a small orbit periodic. The Figures 4-6 illustrated the effectiveness of the control strategy taken to these problems.

\section{References}

[1] I. Horowitz, Survey of quantitative feedback theory (QDT), International Journal of Control, 53, No. 2 (1991), 255-291.

[2] M.A. Dahlel, J.B. Pearson, 11 optimal feedback controllers for MIMO discrete-time system. IEEE Trans. on Auto. Control (1987).

[3] W.J. Rugh, Analytical framework for gain scheduling, IEEE Control System Magazine, 11, No. 1 (1991), 79-84.

[4] P. Apkarian, P. Gabinet, A convex characterization of gain-scheduled H? controllers. IEEE Trans. on Auto. Control, 40, No. 5 (1995), 853-864. 
[5] H.K. Khalil, Nonlinear Systems, New York, Macmillan (1992); C.P. Mracek, J.R. Cloutier, D'C.N. Souza, A new technique for nonlinear estimation, In: Proceedings of the IEEE Conference on Control Applications, Dearborn, MI, September (1996).

[6] E. Ott, C. Grebogi, J.A. Yorque, Controlling Chaos, Phys. Rev. Lett., 66 (1990), 1196.

[7] S.C. Sinha, J.T. Henrichs, B.A. Ravindra, A general approach in the design of active controllers for nonlinear systems exhibiting chaos, Int. J. Bifur. Chaos, 10, No. 1 (2000), 165.

[8] J.R. Coultier, C.N. Souza, C.P. Mracek, Nonlinear regulation and nonlinear $\mathrm{H}$, control via the state-dependent Riccati equation technique; part 1, theory; part 2, examples. In Proceedings of the International Conference on Nonlinear Problems in Aviation and Aerospace. Available through University Press, Embry-Riddle Aeronautical University, Daytona Beach, FL, 32114, May (1996).

[9] C.P. Mracek, J.R. Cloutier, Control designs for the nonlinear benckmark problem via the state-dependent Riccati equation method, International Journal of Robust and Nonlinear Control, 8 (1998), 401-433.

[10] F.R. Chavarette, J.M. Balthazar, N.J. Peruzzi, M. Rafikov, On non-linear dynamics and control designs applied to the ideal and non-ideal variants of the Fitzhugh-Nagumo (FN) mathematical model, Communications in Nonlinear Science and Numerical Simulation, 14, No. 3 (2009), 892-905.

[11] F.R. Chavarette, J.M. Balthazar, J.L.P. Felix, M. Rafikov, A reducing of a chaotic movement to a periodic orbit, of a micro-electro-mechanical system, by using an optimal linear control design, Communications in Nonlinear Science and Numerical Simulation, 14, No. 5 (2009), 1844-1853.

[12] F.R. Chavarette, J.M. Balthazar, J.L.P. Felix, Remarks on an optimal linear control design applied to a nonideal and an ideal structure coupled to an essentially nonlinear oscillator, J. Comput. Nonlinear Dynam., $\mathbf{5}$ (2010), 024501.

[13] F.R. Chavarette, G.R. Campodonio, L. Barbanti, B.C. Damasceno, State dependent Riccati equation control of nonlinear vibrations in a microelectro-mechanical gyroscope system, International Journal of Applied Mathematics, 24 (2011), 131-148. 
[14] A.M. Tusset, J.M. Balthazar, F.R. Chavarette, J.L.P. Felix, On energy transfer phenomena, in a nonlinear ideal and non-ideal essential vibrating systems, coupled to a (MR) magneto-rheological damper, Nonlinear Dynamics, 69 (2012), 1859-1880.

[15] J.D. Pearson, Approximation methods in optimal control, Journal of Electronics and Control, 13 (1962), 453-469.

[16] A. Wernli, G. Cook, Suboptimal control for the nonlinear control design techniques on a model of the Caltech ducted fan, Automatica, 37 (2001), 1971-1978.

[17] B. Friedland, Advanced Control System Design, Prentice-Hall, Englewood Cli, NJ (1996), 110-112.

[18] M. Rafikov, J.M. Balthazar, On control and synchronization in chaotic and hyperchaotic system via linear control feedback, Nonlinear Science and Numerical Simulation, 13 (2008), 1246-1255.

[19] Shih-Yu Li, Sheng-Chieh Huang, Cheng-Hsiung Yang, Zheng-Ming Ge, Generating tri-chaos attractors with three positive Lyapunov exponents in new four order system via linear coupling, Nonlinear Dyn., 69 (2012), 805-816, doi: 10.1007/s11071-011-0306-x.

[20] K.L. Turner, S.A. Miller, P.G. Hartwells, MacN.C. Donald, S.H. Strofatz, S.G. Adams, Five parametric resonances in a microelectromechanical system, Nature, 396 (1998), 149-152. 